\title{
RTC Based Power Control System
}

\author{
Mahesh Pawar, Dnyaneshwari Yelne, Trushita Sonkusare, \\ Samiksha kalamakar, Sandesh Rao, Neeraj Lalwani \\ Department of Electronics Enginnering, Yehswantrao Coleege Of Engineering, Nagpur, Maharashtra, India
}

\begin{abstract}
This research proposes a technology-enhanced alternative to manual switching that is both user-friendly and safe. The ATMEGA328p-based time-controlled electrical device control system takes over the task of turning on and off electrical equipment according to time. It has a built-in real-time clock that tracks the current time. When the real time matches the planned time, the associated device is turned on or off according to the ON/OFF time. Using the rotary encoder, you may change the switching time at any moment. The LCD display shows the current time and the encoded.
\end{abstract}

Keywords: RTC, ATmega328P, LCD, Relay, Rotary encoder, Power Controller.

\section{Introduction}

1. In today's day, more and more factories are adopting automation to replace manual labour. This means different devices/systems interact with each other to complete tasks. In such arrangements there is an absolute need for precision in power ON/OFF time of devices/systems that interact. This is essential for two reasons:

(1) To achieve the desired outcome with smooth transitions and accuracy.

(2) To save power when the services of a particular device are not required

\section{Materials}

\section{ATmega328P}

Based on the AVR® improved RISC architecture, the Atmel® ATmega328P is a low-power CMOS 8-bit microcontroller. The ATmega328P delivers throughputs approaching 1MIPS per MHz by executing strong instructions in a single clock cycle, allowing the system designer to balance power consumption vs processing performance.

The Atmel® ATmega328P has the following features: $32 \mathrm{~K}$ bytes of in-system programmable flash memory, $1 \mathrm{~K}$ bytes EEPROM, 2K bytes SRAM, 23 general-purpose I/O lines, 32 general-purpose working registers, three flexible Timer/Counters with compare modes, internal and external interrupts, a serial programmable

USART, a byte-oriented 2-wire serial interface, an SPI serial port, a 6-channel 10-bit ADC (8 channels in TQFP and QFP), a serial programmable In idle mode, the CPU is shut off, but the SRAM, Timer/Counters,

USART, 2-wire serial interface, SPI port, and interrupt system are still active. 


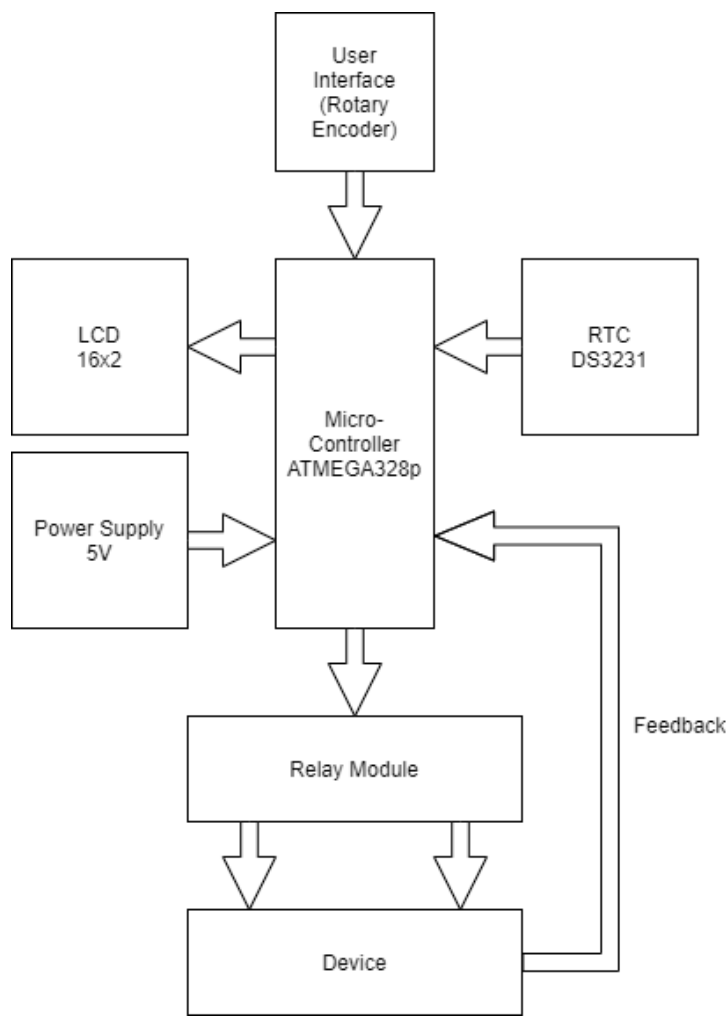

Fig. 1 Block Diagram

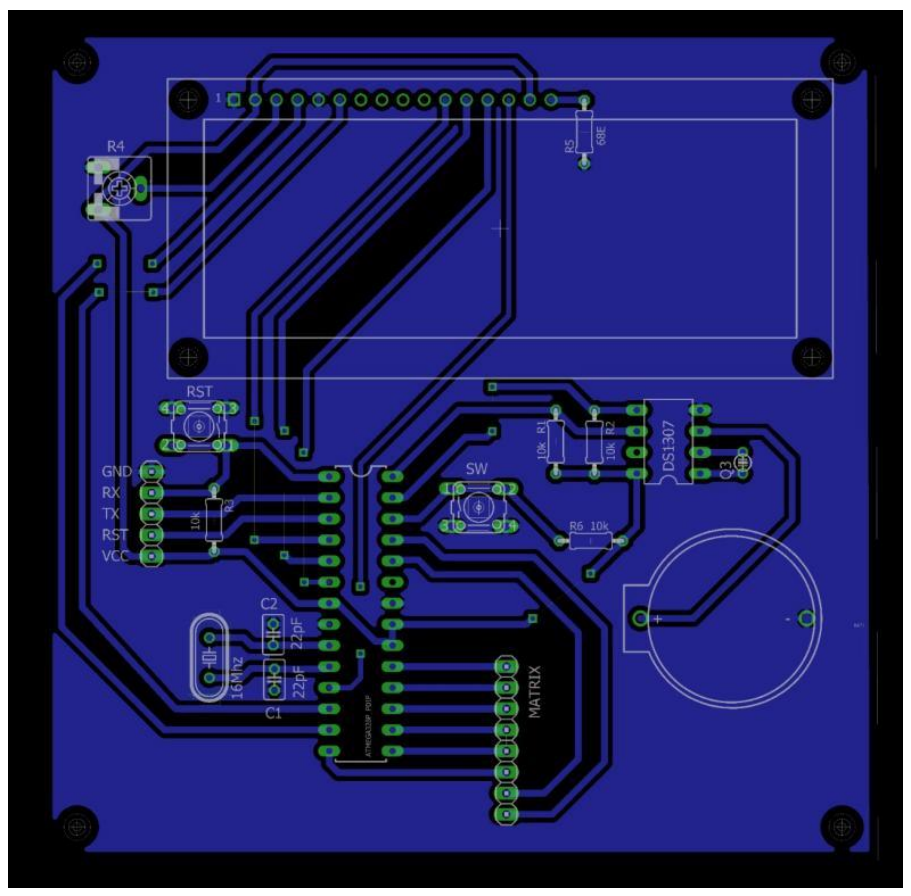

Fig. 2 :- PCB view of Complete Circuit 
All other chip operations are disabled until the next interrupt or hardware reset, as the oscillator is stuck in the power-down state. The oscillator is frozen in the power-down state, which disables all other chip functions until the next interrupt or hardware reset. The asynchronous timer continues to operate in power-saving mode, allowing the user to keep a timer base while the rest of the device sleeps. To reduce switching noise during ADC conversions, the ADC noise reduction mode turns down the CPU and all I/O modules except the asynchronous timer and ADC. The crystal/resonator oscillator runs in standby mode while the rest of the device sleeps. This enables for a very quick start-up while also consuming extremely little power.

\section{RTC DS3231}

The DS3231 is an I2C real-time clock with an integral temperature-compensated crystal oscillator (TCXO) and crystal that is low-cost and exceptionally precise. The instrument has a battery input and keeps accurate timekeeping even when the device's main power is lost. The crystal resonator's inclusion improves the device's long-term precision while also lowering the piece-part count in a production line. The DS3231 comes in a 16-pin, 300-mil SO box and is available in commercial and industrial temperature ranges.

The RTC keeps track of seconds, minutes, hours, days, dates, months, and years. For months with less than 31 days, the date at the end of the month is automatically modified, including leap year corrections. The clock has an AM/PM indication and works in either a 24-hour or 12-hour mode. Two programmable time-of-day alarms are included, as well as a programmable square-wave output. An I2C bidirectional bus is used to transport address and data serially.

\section{LCD Module}

An LCD (Liquid Crystal Display) screen is a type of electronic display that may be used in a variety of ways. A $16 \times 2$ LCD display is a relatively simple module that may be found in a variety of devices and circuits. On each of its two-line segments, a $16 \times 2$ LCD can show 16 characters per line. On this LCD, each character is shown in a 5x7 pixel matrix. The 224 distinct letters and symbols may be shown on the $16 \times 2$ intelligent alphanumeric dot matrix display. The two registers on such LCD are Command and Data.

Various commands sent to the display are stored in command registers. The data register holds the information that will be shown. Putting data that forms the image of what you want to display into the data registers, then commands into the instruction register, is how you control the display. Liquid Crystal Library simplifies this for you in your Arduino project, so you don't have to know the low-level commands. The potentiometer linked across VEE pin can be used to modify the display's contrast.

Specifications:

\begin{tabular}{|l|l|}
\hline Display construction & 16 Characters * 2 Lines \\
\hline Display mode & TN/STN \\
\hline Display type & Positive Transflective \\
\hline Backlight & LED(B/5.0V) \\
\hline Viewing direction & 6 o'clock \\
\hline Driving method & $1 / 16$ duty,1/5 bias \\
\hline Type & COB $($ Chip On Board $)$ \\
\hline Number of data line & 8 -bit parallel \\
\hline Connector & Pin \\
\hline Module size & $80.0 \times 36.0$ \\
\hline Viewing area & $73.8 \times 27.1$ \\
\hline
\end{tabular}

Table 1 :- LCD Specifications 


\section{Relay Module}

The Relay Module is a switch that can control power of electrical circuits with significantly higher potential difference and/or current. A micro-controller cannot tolerate the high potential difference/current footprint of these devices so the relay module is used for the purpose. Relay separates the micro-controller from the high voltage circuit so that the two remain electrically independent. It secures the two circuits from damaging each other. The unit contains 3 connectors - NO, COM, \& NC. The jumper cap can be set to high-level effective or low-level effective mode, according to the input trigger.

\section{Rotary Encoder}

The Keyes KY040 rotary encoder is a rotational input device (as in dial) that indicates both how much and in which direction the dial has been spun. It's an excellent instrument for controlling stepper and servo motors. It might also be used to operate electronic devices such as digital potentiometers. The number of locations per rotation of a rotary encoder is X. As you spin the encoder, these locations are easily sensed as little "clicks." We have thirty of these locations in the Keyes module. Three pins are located on one side of the switch X, Y \& Z. There are two switches within the encoder. Pin X is connected to pin $\mathrm{Z}$ by one switch, while pin $\mathrm{Y}$ is connected to pin $\mathrm{Z}$ by another switch.

These switches alter states as a result of each click:

- If both switches are closed, rotating the encoder one position clockwise or counter clockwise will open both switches.

- If both switches are open, rotating the encoder one position clockwise or counter clockwise closes both switches.

The switch rotates in a clockwise manner if $X$ changed states first.

The switch rotates in a counter clockwise manner if $\mathrm{Y}$ changed states first.

\section{Operation}

Power Turned On. Display shows menu with options to enter "On" hours and minutes and option to save using rotary encoder and button.

After saving “On” hours, display shows menu with options to enter "Off” hours and minutes and option to save.

Once all inputs are saved the MCU compares current time with entered "On" Time and displays current time and time remaining on the display.

When Current time becomes equal to "On" time, signal is given to relay 1 to turn the appliance on.

The circuit for turning off the device has a slight change with ideal state auto turn off.

The device when turned on and operating sends a continuous signal to the MCU.

When the device is not operational the signal stops.

The MCU checks for the signal and when not received starts a timer for 30 mins. This timer resets at every pulse received.

MCU compares current time with entered “Off' time and displays Current time and time remaining on the display.

If the auto-off timer is not interrupted then a signal is given to relay 2 to turn off the appliance. 
Else if the off time becomes equal to current time a signal is given to relay 2 to turn off the appliance.

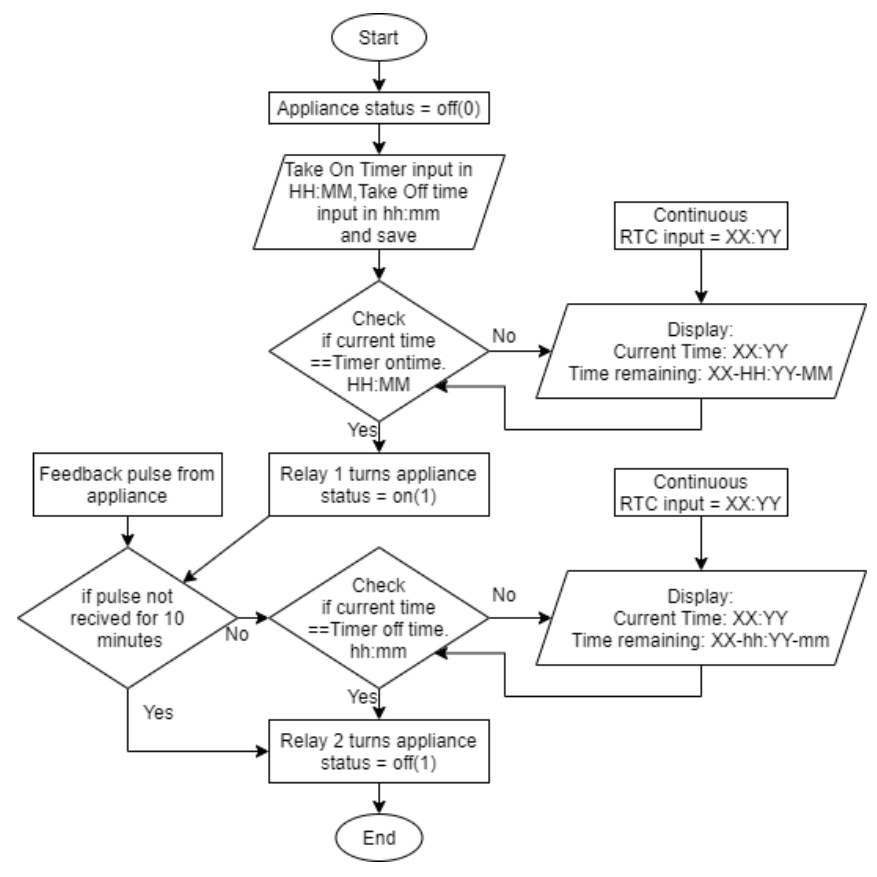

Fig. 3 :- Operations

\section{Result:}

This circuit not only removes the necessity of manual switching but also saves power due to auto-ideal turn off. It can be used to automate unmanned machines and also machines that require pre-emptive start to operate during shifts. This project was made specially for pre-emptive case where a machine requires certain time to become operational. The switching of the machine depends on time so it eliminates presence of humans during off hours. It is used to schedule operating times of the machine and turns it on and renders it operational by the time the shift starts while also minimizing energy wastage with ideal state auto turn off.

\section{Conclusion}

In this paper, the proposed solution is cost efficient and possesses a programable electrical switching arrangement. Time controlled switching system can be used in multiple scenarios of residential, commercial and industrial applications. This solution prevents energy as well as time wastage. 


\section{Future scope}

An array of these devices and remote scheduling can also be implemented to automate assembly lines in production sector. It can also be used in hazardous/dangerous environments where time is crucial and humans cannot be present. Pre-emptive scheduling can also be implemented so that we can queue schedules weeks or months prior.

\section{References}

[1] Awadallah Sulieman Rahama1, Dr. Dalia Mahmoud2, Time Operated Electrical Appliance Controlling Systems by Using Microcontroller, International Journal of Science and Research (IJSR), May 2016

[2] Akkala Lakshmi Durga1, Boodati Prasanthi2, Kandragunta Maheshwari3, Bonthu Greeshma4, TIME OPERATED ELECTRICAL APPIALNES CONTROL SYSTEM USING ARDUINO, International Research Journal of Engineering and Technology (IRJET)

[3] Information and Specifications of ATmega328P from Atmel.com 